TAMKANG JOURNAL OF MATHEMATICS

Volume 33, Number 2, Summer 2002

\title{
NEW BOUNDS FOR SIMPSON'S INEQUALITY
}

\author{
NENAD UJEVIĆ
}

\begin{abstract}
Some new bounds for Simpson's inequality are derived. These bounds are better than some recently obtained bounds.
\end{abstract}

\section{Introduction}

In recent years many authors have written about Simpson's inequality, for example see [1]-[9] and [13]. Simpson's inequality gives an error bound for the well-known Simpson's quadrature rule:

$$
E(f)=\int_{a}^{b} f(t) d t-\frac{b-a}{6}\left[f(a)+4 f\left(\frac{a+b}{2}\right)+f(b)\right] .
$$

There are few known ways to express the term $E(f)$. Different variants of $E(f)$ give different estimations of the error. In this paper we give a new approach to the subject. This new approach is based on a generalization of pre-Grüss inequality which is obtained in [14]. It gives better results.

In [5] we can find the next result.

If we assume that $f^{(n-1)}$ is an absolutely continuous function on $[a, b]$ such that $f^{(n)} \in L_{2}(a, b)(n=1,2,3)$ then we have the Simpson's inequality (for $\left.n \in\{1,2,3\}\right)$,

$$
|E(f)| \leq C_{n}(b-a) \sigma\left(f^{(n)} ; a, b\right),
$$

where $E(f)$ is given by $(1.1)$,

$$
\begin{gathered}
C_{1}=\frac{1}{6}, \quad C_{2}=\frac{1}{12 \sqrt{30}}, \quad C_{3}=\frac{1}{48 \sqrt{105}} \\
\left.\sigma\left(f^{(n)} ; a, b\right)=\left[\frac{1}{b-a}\left\|f^{(n)}\right\|_{2}^{2}-\left(\left[f^{(n-1)} ; a, b\right)\right]\right)^{2}\right]^{1 / 2}
\end{gathered}
$$

and

$$
\left.\left[f^{(n)} ; a, b\right)\right]=\frac{f^{(n)}(b)-f^{(n)}(a)}{b-a}
$$

Received May 02, 2001.

2000 Mathematics Subject Classification. 26D10, 41A55.

Key words and phrases. Pre-Grüss inequality, generalization, Simpson's inequality. 


$$
\left\|f^{(n)}\right\|_{2}^{2}=\int_{a}^{b} f^{(n)}(t)^{2} d t .
$$

The above result is an improvement of a result obtained in [13].

In this paper we give a new expression for the term $E(f)$ and use the generalization of pre-Grüss inequality to derive some better estimations of the error for Simpson's quadrature rule. In fact, we further improve (1.2).

\section{Main Results}

We define the Chebyshev functional:

$$
T(f, g)=\frac{1}{b-a} \int_{a}^{b} f(t) g(t) d t-\frac{1}{(b-a)^{2}} \int_{a}^{b} f(t) d t \int_{a}^{b} g(t) d t
$$

and the functional

$$
\begin{aligned}
S_{\Psi}(f, g)= & \int_{a}^{b} f(t) g(t) d t-\frac{1}{b-a} \int_{a}^{b} f(t) d t \int_{a}^{b} g(t) d t \\
& -\int_{a}^{b} f(t) \Psi_{0}(t) d t \int_{a}^{b} g(t) \Psi_{0}(t) d t,
\end{aligned}
$$

where $f, g, \Psi \in L_{2}(a, b), \Psi_{0}(t)=\Psi(t) /\|\Psi\|_{2}$. We suppose that

$$
\int_{a}^{b} \Psi(t) d t=0
$$

Further, in [11] we can find the pre-Grüss inequality

$$
T(f, g)^{2} \leq T(f, f) T(g, g)
$$

and the Grüss inequality

$$
|T(f, g)| \leq \frac{(\Delta-\delta)(\Gamma-\gamma)}{4},
$$

where $\delta \leq f(t) \leq \Delta$ and $\gamma \leq g(t) \leq \Gamma, t \in[a, b]$. Specially, we have

$$
|T(f, f)| \leq \frac{(\Delta-\delta)^{2}}{4} .
$$

Theorem 1. If $g, h, \Psi \in L_{2}(a, b)$ and (2.3) holds then we have

$$
\left|S_{\Psi}(g, h)\right| \leq S_{\Psi}(g, g)^{1 / 2} S_{\Psi}(h, h)^{1 / 2} .
$$


Proof. We can write

$$
S_{\Psi}(g, h)=\int_{a}^{b} g(t)\left[h(t)-\frac{1}{b-a} \int_{a}^{b} h(s) d s-\int_{a}^{b} h(s) \Psi_{0}(s) d s \Psi_{0}(t)\right] d t .
$$

We also have

$$
\int_{a}^{b}\left[h(t)-\frac{1}{b-a} \int_{a}^{b} h(s) d s-\int_{a}^{b} h(s) \Psi_{0}(s) d s \Psi_{0}(t)\right] d t=0
$$

and

$$
\int_{a}^{b} \Psi_{0}(t)\left[h(t)-\frac{1}{b-a} \int_{a}^{b} h(s) d s-\int_{a}^{b} h(s) \Psi_{0}(s) d s \Psi_{0}(t)\right] d t=0,
$$

since (2.3) holds.

It follows from (2.8)-(2.10) that

$$
\begin{aligned}
S_{\Psi}(g, h)= & \int_{a}^{b}\left[g(t)-\frac{1}{b-a} \int_{a}^{b} g(s) d s-\int_{a}^{b} g(s) \Psi_{0}(s) d s \Psi_{0}(t)\right] \\
& \times\left[h(t)-\frac{1}{b-a} \int_{a}^{b} h(s) d s-\int_{a}^{b} h(s) \Psi_{0}(s) d s \Psi_{0}(t)\right] d t .
\end{aligned}
$$

Then, using (2.11),

$$
S_{\Psi}(g, g)=\int_{a}^{b}\left[g(t)-\frac{1}{b-a} \int_{a}^{b} g(s) d s-\int_{a}^{b} g(s) \Psi_{0}(s) d s \Psi_{0}(t)\right]^{2} d t \geq 0 .
$$

In a similar way we get $S_{\Psi}(h, h) \geq 0$. Now, using the Cauchy inequality and (2.11) we get

$$
\begin{aligned}
\left|S_{\Psi}(g, h)\right| \leq & \left\{\int_{a}^{b}\left[g(t)-\frac{1}{b-a} \int_{a}^{b} g(s) d s-\int_{a}^{b} g(s) \Psi_{0}(s) d s \Psi_{0}(t)\right]^{2} d t\right\}^{1 / 2} \\
& \times\left\{\int_{a}^{b}\left[h(t)-\frac{1}{b-a} \int_{a}^{b} h(s) d s-\int_{a}^{b} h(s) \Psi_{0}(s) d s \Psi_{0}(t)\right]^{2} d t\right\}^{1 / 2} \\
= & S_{\Psi}(g, g)^{1 / 2} S_{\Psi}(h, h)^{1 / 2}
\end{aligned}
$$

This completes the proof.

Remark 1. A more general result can be found in [14]. The mentioned result can be applied in this paper as it is described in [14]. In fact, we here consider only the case $n=1$ of the mentioned result. 
We also have

$$
S_{\Psi}(f, g)=(b-a) T(f, g)-\int_{a}^{b} f(t) \Psi_{0}(t) d t \int_{a}^{b} g(t) \Psi_{0}(t) d t .
$$

Hence, $S_{\Psi}(f, g)$ is a generalization of $T(f, g)$. From (2.13) we easily find that

$$
S_{\Psi}(f, f) \leq(b-a) T(f, f) .
$$

Theorem 2. Let $I \subset R$ be a closed interval and $a, b \in$ Int $I, a<b$.If $f: I \rightarrow R$ is an absolutely continuous function with $f^{\prime} \in L_{2}(a, b)$ then we have

$$
\left|\frac{b-a}{6}\left[f(a)+4 f\left(\frac{a+b}{2}\right)+f(b)\right]-\int_{a}^{b} f(t) d t\right| \leq \frac{(b-a)^{3 / 2}}{6} K_{1},
$$

where

$$
K_{1}=\left[\sigma^{2}\left(f^{\prime} ; a, b\right)(b-a)-\left(\int_{a}^{b} f^{\prime}(t) \Psi_{0}(t) d t\right)^{2}\right]^{1 / 2}
$$

and $\Psi(t)=t-\frac{a+b}{2}$ while $\sigma$ is defined by (1.4).

Proof. We define

$$
p_{1}(t)= \begin{cases}t-a, & t \in\left[a, \frac{a+b}{2}\right] \\ t-b, & t \in\left(\frac{a+b}{2}, b\right] .\end{cases}
$$

It is not difficult to verify that

$$
\int_{a}^{b} p_{1}(t) d t=0
$$

and

$$
\int_{a}^{b} \Psi(t) d t=0
$$

We also have

$$
\begin{aligned}
\left\|p_{1}\right\|_{2}^{2} & =\int_{a}^{b} p_{1}(t)^{2} d t=\frac{(b-a)^{3}}{12} \\
\|\Psi\|_{2}^{2} & =\int_{a}^{b} \Psi(t)^{2} d t=\frac{(b-a)^{3}}{12}
\end{aligned}
$$

such that

$$
\Psi_{0}(t)=\frac{\Psi(t)}{\|\Psi\|_{2}}=\frac{\sqrt{12}}{(b-a)^{3 / 2}}\left(t-\frac{a+b}{2}\right)
$$

and

$$
\int_{a}^{b} p_{1}(x, t) \Psi(t) d t=-\frac{(b-a)^{3}}{24} .
$$


Integrating by parts, we have

$$
\begin{aligned}
\int_{a}^{b} p_{1}(t) f^{\prime}(t) d t & =\int_{a}^{\frac{a+b}{2}}(t-a) f^{\prime}(t) d t+\int_{\frac{a+b}{2}}^{b}(t-b) f^{\prime}(t) d t \\
& =f\left(\frac{a+b}{2}\right)(b-a)-\int_{a}^{b} f(t) d t
\end{aligned}
$$

and

$$
\begin{aligned}
\int_{a}^{b} \Psi(t) f^{\prime}(t) d t & =\int_{a}^{b}\left(t-\frac{a+b}{2}\right) f^{\prime}(t) d t \\
& =\frac{f(a)+f(b)}{2}(b-a)-\int_{a}^{b} f(t) d t .
\end{aligned}
$$

From (2.18) and (2.21)-(2.25) we have

$$
\begin{aligned}
& \int_{a}^{b} p_{1}(t) f^{\prime}(t) d t-\frac{1}{b-a} \int_{a}^{b} p_{1}(t) d t \int_{a}^{b} f^{\prime}(t) d t--\int_{a}^{b} p_{1}(t) \Psi_{0}(t) d t \int_{a}^{b} f^{\prime}(t) \Psi_{0}(t) d t \\
= & f\left(\frac{a+b}{2}\right)(b-a)-\int_{a}^{b} f(t) d t+\frac{1}{2}\left[\frac{f(a)+f(b)}{2}(b-a)-\int_{a}^{b} f(t) d t\right] \\
= & {\left[f\left(\frac{a+b}{2}\right)+\frac{f(a)+f(b)}{4}\right](b-a)-\frac{3}{2} \int_{a}^{b} f(t) d t . }
\end{aligned}
$$

On the other hand, we have

$$
\int_{a}^{b} p_{1}(t) f^{\prime}(t) d t-\frac{1}{b-a} \int_{a}^{b} p_{1}(t) d t \int_{a}^{b} f^{\prime}(t) d t-\int_{a}^{b} p_{1}(t) \Psi_{0}(t) d t \int_{a}^{b} f^{\prime}(t) \Psi_{0}(t) d t=S_{\Psi}\left(p_{1}, f^{\prime}\right) .
$$

From (2.7), (2.26) and (2.27) it follows that

$$
\left|\frac{b-a}{6}\left[f(a)+4 f\left(\frac{a+b}{2}\right)+f(b)\right]-\int_{a}^{b} f(t) d t\right| \leq \frac{2}{3} S_{\Psi}\left(f^{\prime}, f^{\prime}\right)^{1 / 2} S_{\Psi}\left(p_{1}, p_{1}\right)^{1 / 2} .
$$

Using (2.18), (2.20) and (2.23) we get

$$
S_{\Psi}\left(p_{1}, p_{1}\right)=\left\|p_{1}\right\|_{2}^{2}-\frac{1}{b-a}\left(\int_{a}^{b} p_{1}(t) d t\right)^{2}-\left(\int_{a}^{b} p_{1}(t) \Psi_{0}(t) d t\right)^{2}=\frac{(b-a)^{3}}{16} .
$$

We also have

$$
\begin{aligned}
S_{\Psi}\left(f^{\prime}, f^{\prime}\right) & =\left\|f^{\prime}\right\|_{2}^{2}-\frac{1}{b-a}\left(\int_{a}^{b} f^{\prime}(t) d t\right)^{2}-\left(\int_{a}^{b} f^{\prime}(t) \Psi_{0}(t) d t\right)^{2} \\
& =\sigma^{2}\left(f^{\prime} ; a, b\right)(b-a)-\left(\int_{a}^{b} f^{\prime}(t) \Psi_{0}(t) d t\right)^{2}=K_{1}^{2} .
\end{aligned}
$$


From (2.28)-(2.30) we easily get (2.15).

Remark 2. It is obvious that (2.15) is better than the corresponding inequality in (1.2).

Theorem 3. Let $I \subset R$ be a closed interval and $a, b \in$ Int $I, a<b$. If $f: I \rightarrow R$ is such that $f^{\prime}$ is an absolutely continuous function with $f^{\prime \prime} \in L_{2}(a, b)$ then we have

$$
\left|\frac{b-a}{6}\left[f(a)+4 f\left(\frac{a+b}{2}\right)+f(b)\right]-\int_{a}^{b} f(t) d t\right| \leq \frac{(b-a)^{5 / 2}}{12 \sqrt{30}} K_{2},
$$

where

$$
\begin{gathered}
K_{2}=\left[\sigma^{2}\left(f^{\prime \prime} ; a, b\right)(b-a)-\left(\int_{a}^{b} f^{\prime \prime}(t) \Psi_{0}(t) d t\right)^{2}\right]^{1 / 2}, \\
\Psi(t)= \begin{cases}1, & t \in\left[a, \frac{a+b}{2}\right] \\
-1, & t \in\left(\frac{a+b}{2}, b\right]\end{cases}
\end{gathered}
$$

and $\Psi_{0}(t)=\Psi(t) /\|\Psi\|_{2}$.

Proof. We define

$$
p_{2}(t)= \begin{cases}\frac{1}{2}(t-a)\left(t-\frac{2 a+b}{3}\right), & t \in\left[a, \frac{a+b}{2}\right] \\ \frac{1}{2}(t-b)\left(t-\frac{a+2 b}{3}\right), & t \in\left(\frac{a+b}{2}, b\right] .\end{cases}
$$

It is not difficult to verify that

$$
\begin{gathered}
\int_{a}^{b} p_{2}(t) d t=0, \\
\int_{a}^{b} \Psi(t) d t=0 \\
\int_{a}^{b} p_{2}(t) \Psi(t) d t=0 .
\end{gathered}
$$

Integrating by parts, we have

$$
\begin{aligned}
\int_{a}^{b} p_{2}(t) f^{\prime \prime}(t) d t= & \frac{1}{2} \int_{a}^{\frac{a+b}{2}}(t-a)\left(t-\frac{2 a+b}{3}\right) f^{\prime \prime}(t) d t+\frac{1}{2} \int_{\frac{a+b}{2}}^{b}(t-b)\left(t-\frac{a+2 b}{3}\right) f^{\prime \prime}(t) d t \\
= & \frac{(b-a)^{2}}{24} f^{\prime}\left(\frac{a+b}{2}\right)-\frac{(b-a)^{2}}{24} f^{\prime}\left(\frac{a+b}{2}\right)-\int_{a}^{\frac{a+b}{2}}\left(t-\frac{5 a+b}{6}\right) f^{\prime}(t) d t \\
& -\int_{\frac{a+b}{2}}^{b}\left(t-\frac{a+5 b}{6}\right) f^{\prime}(t) d t \\
= & -\frac{b-a}{6}\left[f(a)+4 f\left(\frac{a+b}{2}\right)+f(b)\right]+\int_{a}^{b} f(t) d t .
\end{aligned}
$$


From (2.35), (2.37) and (2.38) it follows that

$$
\begin{aligned}
& \int_{a}^{b} p_{2}(t) f^{\prime \prime}(t) d t-\frac{1}{b-a} \int_{a}^{b} p_{2}(t) d t \int_{a}^{b} f^{\prime \prime}(t) d t-\int_{a}^{b} p_{2}(t) \Psi_{0}(t) d t \int_{a}^{b} f^{\prime \prime}(t) \Psi_{0}(t) d t \\
= & -\frac{b-a}{6}\left[f(a)+4 f\left(\frac{a+b}{2}\right)+f(b)\right]+\int_{a}^{b} f(t) d t .
\end{aligned}
$$

On the other hand, we have

$$
\begin{aligned}
& \int_{a}^{b} p_{2}(t) f^{\prime \prime}(t) d t-\frac{1}{b-a} \int_{a}^{b} p_{2}(t) d t \int_{a}^{b} f^{\prime \prime}(t) d t-\int_{a}^{b} p_{2}(t) \Psi_{0}(t) d t \int_{a}^{b} f^{\prime \prime}(t) \Psi_{0}(t) d t \\
= & S_{\Psi}\left(p_{2}, f^{\prime \prime}\right)
\end{aligned}
$$

Using (2.7), (2.39) and (2.40) we have

$$
\left|\frac{b-a}{6}\left[f(a)+4 f\left(\frac{a+b}{2}\right)+f(b)\right]-\int_{a}^{b} f(t) d t\right| \leq S_{\Psi}\left(p_{2}, p_{2}\right)^{1 / 2} S_{\Psi}\left(f^{\prime \prime}, f^{\prime \prime}\right)^{1 / 2} .
$$

We also have

$$
\begin{aligned}
S_{\Psi}\left(p_{2}, p_{2}\right) & =\left\|p_{2}\right\|_{2}^{2}-\frac{1}{b-a}\left(\int_{a}^{b} p_{2}(t) d t\right)^{2}-\left(\int_{a}^{b} p_{2}(t) \Psi_{0}(t) d t\right)^{2} \\
& =\frac{(b-a)^{5}}{4320}
\end{aligned}
$$

and

$$
S_{\Psi}\left(f^{\prime \prime}, f^{\prime \prime}\right)=\sigma^{2}\left(f^{\prime \prime} ; a, b\right)(b-a)-\left(\int_{a}^{b} f^{\prime \prime}(t) \Psi_{0}(t) d t\right)^{2}=K_{2}^{2} .
$$

From (2.41)-(2.43) we easily get (2.31).

Remark 3. It is obvious that (2.31) is better than the corresponding estimation in (1.2).

Corollary 1. Let the assumptions of Theorem 3 be satisfied. If there exist constants $\gamma, \Gamma \in R$ such that $\gamma \leq f^{\prime \prime}(t) \leq \Gamma, t \in[a, b]$ then we have

$$
\left|\frac{b-a}{6}\left[f(a)+4 f\left(\frac{a+b}{2}\right)+f(b)\right]-\int_{a}^{b} f(t) d t\right| \leq \frac{(b-a)^{3}}{12 \sqrt{30}} K
$$

where

$$
K=\left[\frac{(\Gamma-\gamma)^{2}}{4}-\left(\frac{f^{\prime}(a)-2 f^{\prime}\left(\frac{a+b}{2}\right)+f^{\prime}(b)}{b-a}\right)^{2}\right]^{1 / 2} .
$$


Proof. The proof immediately follows from (2.31)-(2.33) and (2.1), (2.6).

Theorem 4. Let $I \subset R$ be a closed interval and $a, b \in$ Int $I, a<b$. If $f: I \rightarrow R$ is such that $f^{\prime \prime}$ is an absolutely continuous function with $f^{\prime \prime \prime} \in L_{2}(a, b)$ then we have

$$
\left|\frac{b-a}{6}\left[f(a)+4 f\left(\frac{a+b}{2}\right)+f(b)\right]-\int_{a}^{b} f(t) d t\right| \leq \frac{(b-a)^{7 / 2}}{48 \sqrt{105}} K_{3},
$$

where

$$
K_{3}=\left[\sigma^{2}\left(f^{\prime \prime \prime} ; a, b\right)(b-a)-\left(\int_{a}^{b} f^{\prime \prime \prime}(t) \Psi_{0}(t) d t\right)^{2}\right]^{1 / 2}
$$

and

$$
\Psi(t)= \begin{cases}t-\frac{7 a+3 b}{10}, & t \in\left[a, \frac{a+b}{2}\right] \\ t-\frac{3 a+7 b}{10}, & t \in\left(\frac{a+b}{2}, b\right] .\end{cases}
$$

Proof. We define

$$
p_{3}(t)= \begin{cases}\frac{1}{6}(t-a)^{2}\left(t-\frac{a+b}{2}\right), & t \in\left[a, \frac{a+b}{2}\right] \\ \frac{1}{6}(t-b)^{2}\left(t-\frac{a+b}{2}\right), & t \in\left(\frac{a+b}{2}, b\right] .\end{cases}
$$

It is not difficult to verify that

$$
\begin{gathered}
\int_{a}^{b} p_{3}(t) d t=0 \\
\int_{a}^{b} \Psi(t) d t=0 \\
\int_{a}^{b} p_{3}(t) \Psi(t) d t=0 .
\end{gathered}
$$

Integrating by parts, we have

$$
\begin{aligned}
\int_{a}^{b} p_{3}(t) f^{\prime \prime \prime}(t) d t & =\frac{1}{6} \int_{a}^{\frac{a+b}{2}}(t-a)^{2}\left(t-\frac{a+b}{2}\right) f^{\prime \prime \prime}(t) d t+\frac{1}{6} \int_{\frac{a+b}{2}}^{b}(t-b)^{2}\left(t-\frac{a+b}{2}\right) f^{\prime \prime \prime}(t) d t \\
& =-\int_{a}^{b} p_{2}(t) f^{\prime \prime}(t) d t \\
& =\frac{b-a}{6}\left[f(a)+4 f\left(\frac{a+b}{2}\right)+f(b)\right]-\int_{a}^{b} f(t) d t .
\end{aligned}
$$

From (2.50), (2.52) and (2.53) it follows that

$$
\begin{aligned}
& \int_{a}^{b} p_{3}(t) f^{\prime \prime \prime}(t) d t-\frac{1}{b-a} \int_{a}^{b} p_{3}(t) d t \int_{a}^{b} f^{\prime \prime \prime}(t) d t-\int_{a}^{b} p_{3}(t) \Psi_{0}(t) d t \int_{a}^{b} f^{\prime \prime \prime}(t) \Psi_{0}(t) d t \\
= & \frac{b-a}{6}\left[f(a)+4 f\left(\frac{a+b}{2}\right)+f(b)\right]-\int_{a}^{b} f(t) d t .
\end{aligned}
$$


On the other hand, we have

$$
\begin{aligned}
& \int_{a}^{b} p_{3}(t) f^{\prime \prime \prime}(t) d t-\frac{1}{b-a} \int_{a}^{b} p_{3}(t) d t \int_{a}^{b} f^{\prime \prime \prime}(t) d t-\int_{a}^{b} p_{3}(t) \Psi_{0}(t) d t \int_{a}^{b} f^{\prime \prime \prime}(t) \Psi_{0}(t) d t \\
= & S_{\Psi}\left(p_{3}, f^{\prime \prime \prime}\right) .
\end{aligned}
$$

Using (2.54), (2.55) and (2.7) we get

$$
\left|\frac{b-a}{6}\left[f(a)+4 f\left(\frac{a+b}{2}\right)+f(b)\right]-\int_{a}^{b} f(t) d t\right| \leq S_{\Psi}\left(p_{3}, p_{3}\right)^{1 / 2} S_{\Psi}\left(f^{\prime \prime \prime}, f^{\prime \prime \prime}\right)^{1 / 2} .
$$

We also have

$$
\begin{aligned}
S_{\Psi}\left(p_{3}, p_{3}\right) & =\left\|p_{3}\right\|_{2}^{2}-\frac{1}{b-a}\left(\int_{a}^{b} p_{3}(t) d t\right)^{2}-\left(\int_{a}^{b} p_{3}(t) \Psi_{0}(t) d t\right)^{2} \\
& =\frac{(b-a)^{7}}{48 \cdot 48 \cdot 105}
\end{aligned}
$$

and

$$
S_{\Psi}\left(f^{\prime \prime \prime}, f^{\prime \prime \prime}\right)=\sigma^{2}\left(f^{\prime \prime \prime} ; a, b\right)(b-a)-\left(\int_{a}^{b} f^{\prime \prime \prime}(t) \Psi_{0}(t) d t\right)^{2}=K_{3}^{2} .
$$

From (2.56)-(2.58) we easily get (2.46).

Remark 4. It is clear that (2.46) is better than the corresponding estimation in $(1.2)$.

Remark 5. Further improvements of the obtained results are possible. If we really need better error bounds then we can apply the procedure described in this section and a procedure described in [14]. However, some complications may occur - see [14].

\section{References}

[1] P. Cerone, Three points rules in numerical integration, J. Non-linear Analysis, (accepted).

[2] V. Čuljak, C. E. M. Pearce and J. Pečarić, The unified treatment of some inequalities of Ostrowski and Simpson type, (submitted).

[3] S. S. Dragomir, On Simpson's quadrature formula for Lipschitzian mappings and applications, Soochow J. Math. 25 (1999), 175-180.

[4] S. S. Dragomir, On Simpson's quadrature formula for differentiable mappings whose derivatives belong to $L_{p}$ spaces and applications, J. KSIAM, 2 (1999), 49-56.

[5] S. S. Dragomir, Better bounds in some Ostrowski-Grüss type inequalities, RGMIA Research Report Collection 3 Article 3, 2000.

[6] S. S. Dragomir, R. P. Agarwal and P. Cerone, On Simpson's inequality and applications, J. Inequal. Appl. 5(2000), 533-579. 
[7] S. S. Dragomir, P. Cerone and J. Roumeliotis, A new generalization of Ostrowski's integral inequality for mappings whose derivatives are bounded and applications in numerical integration and for special means, Appl. Math. Lett. 13 (2000), 19-25.

[8] S. S. Dragomir, J. Pečarić and S Wang, The unified treatment of trapezoid, Simpson and Ostrowski type inequalities for monotonic mappings and applications, Math. Comput. Modelling 31 (2000), 61-70.

[9] I. Fedotov and S. S. Dragomir, An inequality of Ostrowski type and its applications for Simpson's rule and special means, Math. Inequal. Appl. 2 (1999), 491-499.

[10] A. Ghizzetti and A. Ossicini, Quadrature formulae, Birkhaüses Verlag, Basel/Stuttgart, 1970 .

[11] D. S. Mitrinović, J. E. Pečarić and A. M. Fink, Classical and New Inequalities in Analysis, Kluwer Acad. Publ., Dordrecht/Boston/Lancaster/Tokyo, 1993.

[12] D. S. Mitrinović, J. Pečarić and A. M. Fink, Inequalities involving functions and their integrals and derivatives, Kluwer Acad. Publ., Dordrecht, 1991.

[13] C. E. M. Pearce, J. Pečarić, N. Ujević and S. Varošanec, Generalizations of some inequalities of Ostrowski-Grüss type, Math. Inequal. Appl. 3 (2000), 25-34.

[14] N. Ujević, A generalization of the pre-Grüss inequality and applications, (submitted).

Department of Mathematics, University of Split, Teslina 12/III, 21000 Split, Croatia.

E-mail: ujevic@pmfst.hr 